\title{
Quasigraded Lie Algebras and Modified Toda Field Equations
}

\author{
Taras V. SKRYPNYK † \\ $\dagger$ Bogolyubov Institute for Theoretical Physics, 14-b Metrologichna Str., Kyiv, 03143 Ukraine \\ $¥$ Institute of Mathematics, 3 Tereshchenkivs'ka Str., Kyiv-4, 01601 Ukraine \\ E-mail: tskrypnyk@imath.kiev.ua
}

Received October 31, 2005, in final form March 03, 2006; Published online April 16, 2006

Original article is available at http://www.emis.de/journals/SIGMA/2006/Paper043/

\begin{abstract}
We construct a family of quasigraded Lie algebras that coincide with the deformations of the loop algebras in "principal" gradation and admit Kostant-Adler-Symes scheme. Using them we obtain new Volterra coupled systems and modified Toda field equations for all series of classical matrix Lie algebras $\mathfrak{g}$.
\end{abstract}

Key words: infinite-dimensional Lie algebras; soliton equations

2000 Mathematics Subject Classification: 37K05; 37K30

\section{Introduction}

Integrability of equations of $1+1$ field theory and condensed matter physics is based on the possibility to represent them in the form of the zero-curvature equation $[1,2,3]$ :

$$
\frac{\partial U(x, t, \lambda)}{\partial t}-\frac{\partial V(x, t, \lambda)}{\partial x}+[U(x, t, \lambda), V(x, t, \lambda)]=0
$$

The most productive interpretation of the zero-curvature equation is achieved (see $[3,4,5,6])$ if one treats it as a consistency condition for a set of commuting Hamiltonian flows on a dual space to some infinite-dimensional Lie algebra $\widetilde{\mathfrak{g}}$ of matrix-valued function of $\lambda$ written in the EulerArnold (generalized Lax) form. In this case the corresponding $U-V$ pair coincides with the matrix gradients of mutually commuting Hamiltonians with respect to the natural Lie-Poisson bracket on $\widetilde{\mathfrak{g}}^{*}$. The method that provides the needed set of the commuting Hamiltonian flows is the famous Kostant-Adler scheme [7,3]. The main ingredient of this scheme is an existence of the decomposition of the algebra $\widetilde{\mathfrak{g}}$ into the sum of two subalgebras: $\widetilde{\mathfrak{g}}=\widetilde{\mathfrak{g}}_{+}+\widetilde{\mathfrak{g}}_{-}$.

Such approach was originally based on the graded loop algebras $\widetilde{\mathfrak{g}}=L(\mathfrak{g})=\mathfrak{g} \otimes P\left(\lambda, \lambda^{-1}\right)$ $[3,4,5,6]$ that possess decompositions into sums of two subalgebras. In our previous papers $[8,9,10,11,12]$ it was shown that special quasigraded Lie algebras could be also used in the framework of this approach. More precisely, we have constructed a family of quasigraded Lie algebras $\widetilde{\mathfrak{g}}_{A}$ parameterized by some numerical matrices $A$ possessing the decomposition $\widetilde{\mathfrak{g}}_{A}=\widetilde{\mathfrak{g}}_{A}^{+}+\widetilde{\mathfrak{g}}_{A}^{-1}$. The constructed quasigraded Lie algebras are multiparametric deformations of the loop algebras: $\widetilde{\mathfrak{g}}_{A}$ tends to $L(\mathfrak{g})$ in the limit $A \rightarrow 0$. They also generalize the special elliptic so(3) algebra introduced in the papers [14, 15].

In our previous papers $[12,16,17,18]$, using constructed quasigraded Lie algebras $\widetilde{\mathfrak{g}}_{A}$ we have obtained new hierarchies of integrable equations that coincide with various generalizations of Landau-Lifshitz and anisotropic chiral field hierarchies.

\footnotetext{
${ }^{1}$ Subalgebras isomorphic to $\widetilde{\mathfrak{g}}_{A}^{-}$were independently constructed in [13] as a possible complementary subalgebras to the Lie algebras of Taylor series in the Lie algebra of Laurent power series.
} 
In the present paper we combine our previous results from $[11,16,17,18]$ with ideas of [19] and define a new type of the quasigraded Lie algebras $\widetilde{\mathfrak{g}}_{A}^{\mathrm{pr}}$ admitting Kostant-Adler-Symes scheme and coinciding with deformations of the loop algebras in the principal gradation. More definitely, it turned out that for a special choice of the matrices $A$ (that depends on the classical matrix Lie algebras $\mathfrak{g}$ ) it is possible to define "principal" subalgebras $\widetilde{\mathfrak{g}}_{A}^{\mathrm{pr}} \subset \widetilde{\mathfrak{g}}_{A}$ in the analogous way as for the case of ordinary loop algebras [20,21]. In the case $A \rightarrow 0$ the algebras $\tilde{\mathfrak{g}}_{A}^{\text {pr }}$ coincide with the ordinary loop algebras in the principal gradation.

Using the technique of $[4,5,3,6]$ we develop a general scheme of obtaining of non-linear partial differential equations admitting the zero-curvature representation with the values in the Lie algebras $\widetilde{\mathfrak{g}}_{A}^{\mathrm{pr}}$. For this purpose we explicitly construct dual space, coadjoint action, its invariants and Lie-Poisson brackets for the case of the Lie algebra $\widetilde{\mathfrak{g}}_{A}^{\mathrm{pr}}$. In the result we obtain new integrable hierarchies satisfying zero-curvature conditions associated with the Lie algebras $\widetilde{\mathfrak{g}}_{A}^{\mathrm{pr}}$. We show that among the corresponding equations there are remarkably simple partial differential equations which we call "generalized Volterra coupled systems". They have the following form:

$$
\partial_{t} u_{i}=u_{i} \beta_{i}^{A}(v), \quad \partial_{x} v_{i}=v_{i} \alpha_{i}(u),
$$

where $\beta_{i}^{A}$ are linear forms ("pseudo-roots") on the subspace $\mathfrak{g}_{-1}$ depending on the "deformation" matrix $A$ and Lie algebra $\mathfrak{g}, \alpha_{i} \in \Pi \bigcup\{-\Theta\}$ are the linear forms (roots) on the Cartan subalgebra $\mathfrak{g}_{\overline{0}}=\mathfrak{h}, H_{i}$ is the basis in $\mathfrak{h}, E_{-\alpha_{i}}, E_{\Theta}$ is the basis in the subspace $\mathfrak{g}_{-1}$ and

$$
U=u \equiv \sum_{i=1}^{\operatorname{dim} \mathfrak{h}} u_{i} H_{i}, \quad V=\lambda^{-1} v \equiv \lambda^{-1} \sum_{\alpha_{i} \in \Pi \bigcup \Theta} v_{i} E_{-\alpha_{i}},
$$

is the $U-V$-pair for the corresponding "deformed" zero-curvature conditions.

It is necessary to notice that in the case $\mathfrak{g}=g l(n)$ our equations (1) coincide with the periodic closure of the infinite Volterra coupled system considered in [22]. In the non-periodic $g l(n)$ case it was also considered in [23]. For the case of the other series of classical Lie algebras equations (1) seem to be new.

Using parametrization generalizing one from [22] equations (1)could be re-written in the form of the "modified Toda-field equations":

$$
\partial_{x t}^{2} \psi_{i}=\partial_{x} \psi_{i}\left(\sum_{\alpha_{j} \in \Pi \cup\{-\Theta\}} \beta_{i, j}^{A} e^{\alpha_{j}(\psi)}\right)
$$

The structure of the present article is as follows: in the second section we define the quasigraded Lie algebras $\widetilde{\mathfrak{g}}_{A}$ and their "principal" subalgebras. In the third section we define dual spaces, Lie-Poisson brackets and Casimir functions on $\widetilde{\mathfrak{g}}_{A}^{\mathrm{pr}}$. In the fourth section we obtain the zero-curvature equations with the values in the twisted subalgebras $\widetilde{\mathfrak{g}}_{A}^{\mathrm{pr}}$ and consider the case of modified Toda field hierarchies as the main examples they yield.

\section{$2 \quad$ Principal grading of simple Lie algebras}

In this subsection we will introduce necessary notations and remind some important facts from the theory of semisimple Lie algebras $[20,21]$. Let algebra $\mathfrak{g}$ with the bracket $[\cdot, \cdot]$ be a simple (reductive) classical Lie algebra of the rank $n$. Let $\mathfrak{h} \subset \mathfrak{g}$ be its Cartan subalgebra, $\Delta_{ \pm}$be its set of positive(negative) roots, $\Pi$ - the set of simple roots, $H_{i} \in \mathfrak{h}$ the basis of Cartan subalgebra $E_{\alpha}$, $\alpha \in \Delta$ the corresponding root vectors. 
Let us define the so-called "principal" grading of $\mathfrak{g}[20]$, putting:

$$
\operatorname{deg} H_{i}=0, \quad \operatorname{deg} E_{\alpha_{i}}=1, \quad \operatorname{deg} E_{-\alpha_{i}}=-1 .
$$

It is evident that in such a way we obtain the grading of $\mathfrak{g}: \mathfrak{g}=\sum_{k=0}^{h-1} \mathfrak{g}_{\bar{k}}$ with the graded subspaces $\mathfrak{g}_{\bar{k}}$ defined as follows: $\mathfrak{g}_{\bar{k}}=\operatorname{Span}_{C}\left\{E_{\alpha}\right\}$, where $\alpha$ is the root of the height $k$, i.e. $\alpha=\sum_{i=1}^{r} k_{i} E_{\alpha_{i}}$ if $\alpha \in \Delta_{+}, \alpha=\sum_{i=1}^{r} k_{i} E_{-\alpha_{i}}$ if $\alpha \in \Delta_{-}$and $k=\sum_{i=1}^{r} k_{i}, h$ is the Coxeter number of $\mathfrak{g}$. In particular $\mathfrak{g}_{\overline{0}}=\mathfrak{h}, \mathfrak{g}_{\overline{1}}=\operatorname{Span}_{C}\left\{E_{\alpha_{i}}, E_{-\theta} \mid \alpha_{i} \in \Pi\right\}, \mathfrak{g}_{-1}=\operatorname{Span}_{C}\left\{E_{-\alpha_{i}}, E_{\theta} \mid \alpha_{i} \in \Pi\right\}$ and $\theta$ is the highest root of the height $h-1$.

Let us consider the following examples of classical matrix Lie algebras:

Example 1. Let $\mathfrak{g}=g l(n)$ with the basis $\left(X_{i j}\right)_{a b}=\delta_{i a} \delta_{j b}, i, j \in 1, n$ and the standard commutation relations:

$$
\left[X_{i j}, X_{k l}\right]=\delta_{k j} X_{i l}-\delta_{i l} X_{k j} .
$$

In this case $\mathfrak{g}_{1}=\operatorname{Span}_{C}\left\{E_{\alpha_{i}} \equiv X_{i i+1}, E_{-\theta} \equiv X_{n 1} \mid i \in 1, n-1\right\}, \mathfrak{g}_{0}=\operatorname{Span}_{C}\left\{H_{i} \equiv X_{i i} \mid i \in 1, n\right\}$, $\mathfrak{g}_{-1}=\operatorname{Span}_{C}\left\{E_{-\alpha_{i}} \equiv X_{i+1,1}, E_{\theta} \equiv X_{1 n} \mid i \in 1, n-1\right\}$ and the Coxeter number is $h=n$.

Example 2. Let $\mathfrak{g}=s o(2 n+1)$, where $s o(2 n+1)=\left\{X \in g l(2 n+1) \mid X=-s X^{\top} s\right\}$ and $s=\operatorname{diag}\left(1, s_{2 n}\right), s_{2 n}=\left(\begin{array}{cc}0 & 1_{n} \\ 1_{n} & 0\end{array}\right)$. In such realization the Cartan subalgebra has a basis $H_{i}=X_{i+1, i+1}-X_{i+n+1, i+n+1}$ where $i=1, n$, generators of algebra that correspond to the simple roots are $E_{\alpha_{i}}=X_{i+1, i+2}-X_{n+i+2, n+i+1}, i=1, n-1, E_{\alpha_{n}}=X_{n+1,1}-X_{1,2 n+1}$, their negative counterparts are $E_{-\alpha_{i}}=X_{i+2, i+1}-X_{n+i+1, n+i+2}, i=1, n-1, E_{-\alpha_{n}}=X_{1,1+n}-X_{2 n+1,1}$. The highest root corresponds to $E_{\theta}=X_{32+n}-X_{23+n}$, its negative counterpart corresponds to $E_{-\theta}=X_{2+n 3}-X_{3+n 2}$, the Coxeter number is $h=2 n$.

Example 3. Let $\mathfrak{g}=s p(n)$, where $s p(n)=\left\{X \in g l(2 n) \mid X=w X^{\top} w\right\}$ and $w=\left(\begin{array}{cc}0 & 1_{n} \\ -1_{n} & 0\end{array}\right)$. In such realization the Cartan subalgebra has a basis $H_{i}=X_{i, i}-X_{i+n, i+n}$ where $i=1, n$, generators of algebra that correspond to the simple roots are $E_{\alpha_{i}}=X_{i, i+1}-X_{n+i+1, n+i}, i=1, n-1$, $E_{\alpha_{n}}=X_{n, 2 n}$, their negative counterparts are $E_{-\alpha_{i}}=X_{i+1, i}-X_{n+i, n+i+1}, i=1, n-1, E_{-\alpha_{n}}=$ $X_{2 n, n}$. The highest root corresponds to $E_{\theta}=X_{11+n}$, its negative counterpart corresponds to $E_{-\theta}=X_{1+n 1}$, the Coxeter number is $h=2 n$.

Example 4. Let $\mathfrak{g}=s o(2 n)$, where $s o(2 n)=\left\{X \in g l(2 n) \mid X=-s X^{\top} s\right\}$ and $s \equiv s_{2 n}, s_{2 n}=$ $\left(\begin{array}{cc}0 & 1_{n} \\ 1_{n} & 0\end{array}\right)$. In such realization the Cartan subalgebra has a basis $H_{i}=X_{i, i}-X_{i+n, i+n}$ where $i=1, n$, generators of algebra that correspond to the simple roots are $E_{\alpha_{i}}=X_{i, i+1}-X_{n+i+1, n+i}$, $i=1, n-1, E_{\alpha_{n}}=X_{n, 2 n-1}-X_{n-1,2 n}$, their negative counterparts are $E_{-\alpha_{i}}=X_{i+1, i}-X_{n+i, n+i+1}$, $i=1, n-1, E_{-\alpha_{n}}=X_{2 n-1, n}-X_{2 n, n-1}$. The highest root corresponds to $E_{\theta}=X_{21+n}-X_{12+n}$, its negative counterpart corresponds to $E_{-\theta}=X_{1+n 2}-X_{2+n 1}$, the Coxeter number is $h=2 n-2$.

\section{3 "Principal" quasigraded Lie algebras}

\subsection{General case}

It is well-known [20] that having the "principal" grading of $\mathfrak{g}$ it is possible to define corresponding grading of loop space. Let $\mathfrak{g}=\sum_{k=0}^{h-1} \mathfrak{g}_{\bar{k}}$ be the $\mathbb{Z} / h \mathbb{Z}$ grading of $\mathfrak{g}$. Let us consider the subspace 
$\widetilde{\mathfrak{g}}^{\mathrm{pr}} \subset \widetilde{\mathfrak{g}}$, where $\widetilde{\mathfrak{g}} \equiv \mathfrak{g} \otimes P\left(\lambda, \lambda^{-1}\right)$ of the following type:

$$
\widetilde{\mathfrak{g}}^{\mathrm{pr}}=\bigoplus_{j \in \mathbb{Z}} \mathfrak{g}_{j} \otimes \lambda^{j}
$$

Here $\bar{j}$ denotes the class of equivalence of the elements $j \in \mathbb{Z} \bmod h \mathbb{Z}$. From the fact that $\left[\mathfrak{g}_{\bar{i}}, \mathfrak{g}_{\bar{j}}\right] \subset \mathfrak{g}_{\overline{i+j}}$ it follows that $\widetilde{\mathfrak{g}}^{\mathrm{pr}}$ is a closed Lie algebra with respect to the ordinary Lie bracket on the tensor product:

$$
[X \otimes p(\lambda), Y \otimes q(\lambda)]=[X, Y] \otimes p(\lambda) q(\lambda)
$$

where $X \otimes p(\lambda), Y \otimes q(\lambda) \in \widetilde{\mathfrak{g}}^{\text {pr }}$. It is evident from the definition itself that $\widetilde{\mathfrak{g}}^{\text {pr }}$ is the graded Lie algebra with the grading being defined by the degrees of the spectral parameter $\lambda$.

Let us introduce the structure of the quasigraded Lie algebra into the loop space $\widetilde{\mathfrak{g}}$. In order to do this we will deform Lie algebraic structure in loop algebras $\widetilde{\mathfrak{g}}$ in the following way $[11,12,13,14,15,16,17]$ :

$$
[X \otimes p(\lambda), Y \otimes q(\lambda)]_{F}=[X, Y] \otimes p(\lambda) q(\lambda)-F(X, Y) \otimes \lambda p(\lambda) q(\lambda),
$$

where $X \otimes p(\lambda), Y \otimes q(\lambda) \in \widetilde{\mathfrak{g}}$ and the map $F: \mathfrak{g} \times \mathfrak{g} \rightarrow \mathfrak{g}$ is skew and satisfies the following two requirements which are equivalent to the Jacobi identities:

$$
\begin{aligned}
& \sum_{\text {c.p. }\{i, j, k\}}\left(F\left(\left[X_{i}, X_{j}\right], X_{k}\right)+\left[F\left(X_{i}, X_{j}\right), X_{k}\right]\right)=0, \\
& \sum_{\text {c.p. }\{i, j, k\}} F\left(F\left(X_{i}, X_{j}\right), X_{k}\right)=0 .
\end{aligned}
$$

Now we are interested in a possibility of defining the structure of the quasigraded algebra on the space $\widetilde{\mathfrak{g}}^{\mathrm{pr}}$. For this purpose we want bracket (2) to be correctly restricted to the space $\widetilde{\mathfrak{g}}^{\mathrm{pr}}$. By the direct verification one can prove the following proposition:

Proposition 1. The subspace $\widetilde{\mathfrak{g}}^{\text {pr }} \subset \widetilde{\mathfrak{g}}$ is the closed Lie algebra if and only if:

$$
F\left(\mathfrak{g}_{\bar{i}}, \mathfrak{g}_{\bar{j}}\right) \subset \mathfrak{g}_{i+j+1} .
$$

In the next subsection we will present examples of the cocycles $F$ on the finite-dimensional Lie algebras $\mathfrak{g}$ that satisfy conditions (J1), (J2) and (3).

\subsection{Case of classical matrix Lie algebras}

Let us now consider the classical matrix Lie algebras $\mathfrak{g}$ of the type $g l(n), s o(n)$ and $s p(n)$ over the field $\mathbb{K}$ of the complex or real numbers. As in the above examples we will realize the algebra $s o(n)$ as algebra of skew-symmetric matrices: $s o(n)=\left\{X \in g l(n) \mid X=-s X^{\top} s\right\}$ and the algebra $s p(n)$ as the following matrix Lie algebra: $s p(n)=\left\{X \in g l(2 n) \mid X=w X^{\top} w\right\}$, where $s \in \operatorname{symm}(n), s^{2}=1, w \in s o(2 n)$ and $w^{2}=-1$.

Let us consider the cochain $F: \mathfrak{g} \times \mathfrak{g} \rightarrow \mathfrak{g}$ of the following form:

$$
F_{A}(X, Y)=[X, Y]_{A} \equiv X A Y-Y A X
$$

From the theory of consistent Poisson brackets it is known [25] to satisfy conditions (J1), (J2).

The following Proposition holds true: 
Proposition 2. The cochain $F_{A}$ satisfies condition (3) if and only if the matrix $A$ has the form:
1) $A=\sum_{i=1}^{n-1} a_{i} X_{i i+1}+a_{n} X_{n 1} \quad$ if $\quad \mathfrak{g}=g l(n)$;
2) $A=\sum_{i=1}^{n-1} a_{i}\left(X_{i+1, i+2}+X_{n+i+2, n+i+1}\right)+a_{n}\left(X_{1+n, 1}+X_{1,2 n+1}\right)+a_{n+1}\left(X_{2+n 3}+X_{3+n 2}\right)$
if $\mathfrak{g}=s o(2 n+1)$;
3) $\quad A=\sum_{i=1}^{n-1} a_{i}\left(X_{i, i+1}+X_{n+i+1, n+i}\right) \quad$ if $\quad \mathfrak{g}=s p(n)$;
4) $A=\sum_{i=1}^{n-1} a_{i}\left(X_{i, i+1}+X_{n+i+1, n+i}\right)+a_{n}\left(X_{n, 2 n-1}+X_{n-1,2 n}\right)+a_{n+1}\left(X_{1+n 2}+X_{2+n 1}\right)$
if $\mathfrak{g}=s o(2 n)$,

where $X_{i j}$ is the standard matrix basis of $g l(n),\left(X_{i j}\right)_{\alpha \beta}=\delta_{i \alpha} \delta_{\gamma \beta}$.

Proof. For the bracket constructed with the help of the cocycle $F_{A}$ to be correctly defined on $\widetilde{\mathfrak{g}}^{\text {pr }}$ we have to require the linear space $\mathfrak{g}$ to be closed with respect to the bracket $[\cdot, \cdot]_{A}$ and linear space $\widetilde{\mathfrak{g}}^{\mathrm{pr}}$ as a space of matrix-valued function of $\lambda$ to be closed with respect to the bracket (4). These conditions are equivalent to the following requirement: $[X, Y]_{A} \in \mathfrak{g}_{i+j+1}$ $\forall X \in \mathfrak{g}_{i}, Y \in \mathfrak{g}_{j}$. The straightforward verification shows in each case that this requirement is satisfied if and only if matrix $A$ has the form described in the proposition.

Hence in the case of the matrix Lie algebras and the matrices $A$ defined in the above Proposition we may introduce into the space $\widetilde{\mathfrak{g}}^{\mathrm{pr}}$ the new Lie bracket of the form:

$$
[X \otimes p(\lambda), Y \otimes q(\lambda)]=[X, Y] \otimes p(\lambda) q(\lambda)-[X, Y]_{A} \otimes \lambda p(\lambda) q(\lambda)
$$

where $X \otimes p(\lambda), Y \otimes q(\lambda) \in \widetilde{\mathfrak{g}}^{\mathrm{pr}},[X, Y] \equiv X Y-Y X$ in the righthand side of this identity denote ordinary Lie bracket in $\mathfrak{g}$ and $[X, Y]_{A} \equiv X A Y-Y A X$.

Definition. We will denote the linear space $\widetilde{\mathfrak{g}}^{\mathrm{pr}}$ with the bracket given by (4) by $\widetilde{\mathfrak{g}}_{A}^{\mathrm{pr}}$.

From the very definition of $\widetilde{\mathfrak{g}}_{A}^{\mathrm{pr}}$ it follows that the algebra $\widetilde{\mathfrak{g}}_{A}^{\mathrm{pr}}$ is $Z$-quasigraded and $\widetilde{\mathfrak{g}}_{A}^{\mathrm{pr}}$ admits the direct sum decomposition $\widetilde{\mathfrak{g}}_{A}^{\mathrm{pr}}=\widetilde{\mathfrak{g}}_{A}^{\mathrm{pr}+}+\widetilde{\mathfrak{g}}_{A}^{\mathrm{pr}-}$, where

$$
\widetilde{\mathfrak{g}}_{A}^{\mathrm{pr}+}=\bigoplus_{j \geq 0} \mathfrak{g}_{j} \otimes \lambda^{j}, \quad \widetilde{\mathfrak{g}}_{A}^{\mathrm{pr}-}=\bigoplus_{j<0} \mathfrak{g}_{j} \otimes \lambda^{j}
$$

In the subsequent exposition we will also use the following statement:

Proposition 3. For all the above classical matrix Lie algebras $\mathfrak{g}$ and matrices $A$ defined in the Proposition 2 it is possible to introduce the following "pseudo-roots" $\beta_{i}^{A}$ :

$$
\left[X, H_{i}\right]_{A}=\beta_{i}^{A}(X) H_{i}
$$

where $X \in \mathfrak{g}_{-1}$ and $H_{i}$ are basic elements in Cartan subalgebra defined in the Section 2.

Remark 1. We call linear forms $\beta_{i}^{A}$ to be "pseudo-roots" because $\mathfrak{g}_{A}=(\mathfrak{g},[\cdot, \cdot])$ in the general case is not isomorphic to $\mathfrak{g}$ and $\mathfrak{g}_{-1} \equiv\left(\mathfrak{g}_{A}\right)_{\overline{0}}$ is not commutative with respect to the $[\cdot, \cdot]_{A}$-bracket. 


\section{Dual space and Lie-Poisson bracket}

In order to describe applications of the Lie algebras $\widetilde{\mathfrak{g}}_{A}^{\mathrm{pr}}$ to the theory of finite-dimensional integrable Hamiltonian systems it is necessary to define linear space $\left(\widetilde{\mathfrak{g}}_{A}^{\mathrm{pr}}\right)^{*}$, the corresponding Lie-Poisson bracket and the Casimir functions of $\widetilde{\mathfrak{g}}_{A}^{\mathrm{pr}}$.

\subsection{Coadjoint representation and invariant functions of $\widetilde{\mathfrak{g}}_{A}^{\mathrm{pr}}$}

In this subsection we will construct the dual space, coadjoint representation and its invariants for the case of the "principal" quasigraded Lie algebras $\widetilde{\mathfrak{g}}_{A}^{\mathrm{pr}}$. If $h$ is the Coxeter number then from properties of invariant form on simple Lie algebras it follows [20] that $\left(\mathfrak{g}_{\bar{i}}, \mathfrak{g}_{\bar{j}}\right)=0$ if $i+j \neq 0$ $\bmod h$. Hence we can define pairing between $\widetilde{\mathfrak{g}}_{A}^{\mathrm{pr}}$ and $\left(\widetilde{\mathfrak{g}}_{A}^{\mathrm{pr}}\right)^{*}$ in the following way:

$$
\langle X(\lambda), L(\lambda)\rangle=\operatorname{res}_{\lambda=0} \lambda^{-1} \operatorname{Tr}(X(\lambda) L(\lambda))
$$

From this definition it follows that the generic element $L(\lambda) \in\left(\widetilde{\mathfrak{g}}_{A}^{\mathrm{pr}}\right)^{*}$ has the form:

$$
L(\lambda)=\sum_{j \in Z} \sum_{\alpha=1}^{\operatorname{dim} \mathfrak{g}_{\bar{j}}} l_{\alpha}^{(j)} X_{\alpha}^{\overline{-j}} \lambda^{-j},
$$

where $X_{\alpha}^{\overline{-j}}$ is an element of basis of subspace $\mathfrak{g}_{\overline{-j}}, l_{\alpha}^{(j)}$ is a coordinate function on $\left(\widetilde{\mathfrak{g}}_{A}^{\mathrm{pr}}\right)^{*}$.

Remark 2. We use the notion of infinite-dimensional Lie algebras $\widetilde{\mathfrak{g}}_{A}$ and $\widetilde{\mathfrak{g}}_{A}^{\mathrm{pr}}$ in the algebraic sense of [20], i.e. we require that each of its elements consists of finite linear combination of an infinite set of the natural elements of basis $\left\{X_{\alpha}^{\bar{j}} \lambda^{j}\right\}$. On the contrary, we let the dual space $\widetilde{\mathfrak{g}}^{*}$ to be wider, i.e. to contain also infinite linear combination of the elements of its basis $\left(X_{\alpha}^{\bar{j}} \lambda^{j}\right)^{*}=X_{\alpha}^{\overline{-j}} \lambda^{-j}$. Under such agreement all subsequent consideration will be correct and consistent (see [18]).

The following proposition holds true:

Proposition 4. Let $L(\lambda) \in\left(\widetilde{\mathfrak{g}}_{A}^{\mathrm{pr}}\right)^{*}$ be the generic element of the dual space. Then the functions

$$
I_{k}^{m}(L(\lambda))=\frac{1}{m} \operatorname{res}_{\lambda=0} \lambda^{-k-1} \operatorname{Tr}\left(L(\lambda) A(\lambda)^{-1}\right)^{m},
$$

are invariants of the coadjoint representation of the Lie algebra $\widetilde{\mathfrak{g}}_{A}^{\mathrm{pr}}$

Proof. It follows from the explicit form of the coadjoint action which has the following form:

$$
\operatorname{ad}_{X(\lambda)}^{*} \circ L(\lambda)=A(\lambda) X(\lambda) L(\lambda)-L(\lambda) X(\lambda) A(\lambda)
$$

where $A(\lambda)=(1-\lambda A), X(\lambda), Y(\lambda) \in \widetilde{\mathfrak{g}}_{A}^{\mathrm{pr}}, L(\lambda) \in\left(\widetilde{\mathfrak{g}}_{A}^{\mathrm{pr}}\right)^{*}$.

Remark 3. The matrix $A(\lambda)^{-1} \equiv(1-\lambda A)^{-1}$ in the above Proposition has to be understood as a power series in $\lambda$ in the neighborhood of 0 or $\infty$ : $A(\lambda)^{-1}=\left(1+A \lambda+A^{2} \lambda^{2}+\cdots\right)$ or $A(\lambda)^{-1}=-\left(A^{-1} \lambda^{-1}+A^{-2} \lambda^{-2}+\cdots\right)$. The corresponding decomposition should be chosen in such a way that the restriction of the invariant function $I_{k}^{m}(L(\lambda))$ onto the dual space $\left(\widetilde{\mathfrak{g}}_{A}^{\mathrm{pr} \pm}\right)^{*}$ are finite polynomials (see [18]). More precisely, when restricting invariant functions on $\left(\widetilde{\mathfrak{g}}_{A}^{\mathrm{pr}-}\right)^{*}$ one has to choose the decomposition of $A(\lambda)^{-1}$ in the neighborhood of 0 , and when restricting invariant function on $\left(\widetilde{\mathfrak{g}}_{A}^{\mathrm{pr}+}\right)^{*}$ one has to chose the decomposition of $A(\lambda)^{-1}$ in the neighborhood of $\infty$. 


\subsection{Lie-Poisson bracket}

Let us define Poisson structures in the space $\left(\widetilde{\mathfrak{g}}_{A}^{\mathrm{pr}}\right)^{*}$. Using pairing (6) (described in the previous section) we can define Lie-Poisson bracket on $P\left(\left(\widetilde{\mathfrak{g}}_{A}^{\mathrm{pr}}\right)^{*}\right)$ in the standard way:

$$
\left\{F_{1}(L(\lambda)), F_{2}(L(\lambda))\right\}=\left\langle L(\lambda),\left[\nabla F_{1}(L(\lambda)), \nabla F_{2}(L(\lambda))\right]_{A(\lambda)}\right\rangle,
$$

here $\nabla F_{i}(L(\lambda))=\sum_{j \in Z} \sum_{\alpha=1}^{\operatorname{dim} \mathfrak{g}_{\bar{j}}} \frac{\partial F_{i}}{\partial l_{\alpha}^{(j)}} X_{\alpha}^{\bar{j}} \lambda^{j}$ and $X_{\alpha}^{\bar{j}}$ is an element of basis of subspace $\mathfrak{g}_{\bar{j}}$.

From the Proposition 4 and standard arguments follows the next corollary:

Corollary 1. The functions $I_{k}^{m}(L(\lambda))$ are central (Casimir) functions for the Lie-Poisson bracket (7).

Let us calculate Poisson bracket (7) explicitly. It is easy to show that for the coordinate functions $l_{\alpha}^{(i)}, l_{\beta}^{(j)}$, where $l_{\alpha}^{(i)} \in\left(\mathfrak{g}_{\bar{i}}\right)^{*}, l_{\beta}^{(j)} \in\left(\mathfrak{g}_{j}\right)^{*}$, this bracket will have the following form:

$$
\left\{l_{\alpha}^{(i)}, l_{\beta}^{(j)}\right\}=\sum_{\gamma} C_{\alpha, \beta}^{\gamma} l_{\gamma}^{(i+j)}-\sum_{\delta} C_{\alpha, \beta}^{\delta}(A) l_{\delta}^{(i+j+1)}
$$

where $l_{\gamma}$ and $l_{\delta}$ are the coordinate functions on $\left(\mathfrak{g}_{\overline{i+j}}\right)^{*}$ and $\left(\mathfrak{g}_{\overline{i+j+1}}\right)^{*}$. This bracket determines the Lie algebra structure isomorphic to $\widetilde{\mathfrak{g}}_{A}^{\mathrm{pr}}$ in the space of linear functions $\left\{l_{\alpha}^{i}\right\}$ and, hence, subspaces $\left(\left(\widetilde{\mathfrak{g}}_{A}^{\mathrm{pr}}\right)^{ \pm}\right)^{*}$ are Poisson.

\subsection{Infinite-component Hamiltonian systems via $\widetilde{\mathfrak{g}}_{A}^{\mathrm{pr}}$}

In this subsection we construct Hamiltonian systems on the infinite-dimensional space $\left(\widetilde{\mathfrak{g}}_{A}^{\mathrm{pr}}\right)^{*}$ possessing infinite number of independent, mutually commuting integrals of motion. Let

$$
L^{\mp}(\lambda)=\sum_{j \in \mathbb{Z}_{ \pm}} \sum_{\alpha=1}^{\operatorname{dim} \mathfrak{g}_{\bar{j}}} L_{\alpha}(\lambda) X_{\alpha}^{\overline{-j}}=\sum_{j \in \mathbb{Z}_{ \pm}} \sum_{\alpha=1}^{\operatorname{dim} \mathfrak{g}_{\bar{j}}} l_{\alpha}^{(j)} \lambda^{-j} X_{\alpha}^{\overline{-j}}
$$

be the generic elements of the spaces $\left(\widetilde{\mathfrak{g}}_{A}^{\mathrm{pr} \pm}\right)^{*}$. Let us consider the functions on $\left(\widetilde{\mathfrak{g}}_{A}^{\mathrm{pr}}\right)^{*}$ of the form

$$
I_{k}^{m \mp}(L(\lambda)) \equiv I_{k}^{m}\left(L^{\mp}(\lambda)\right)
$$

where $\left\{I_{k}^{m}(L(\lambda))\right\}$ are Casimir functions of $\tilde{\mathfrak{g}}_{A}^{\mathrm{pr}}$. Due to the Remark 3 functions $I_{k}^{m \mp}(L(\lambda))$ are finite polynomials on $\left(\widetilde{\mathfrak{g}}_{A}^{\mathrm{pr}}\right)^{*}$. The Hamiltonian flows corresponding to the Hamiltonians $I_{k}^{m \mp}(L(\lambda))$ are written in a standard way:

$$
\frac{\partial L_{\alpha}(\lambda)}{\partial t_{k}^{m \mp}}=\left\{L_{\alpha}(\lambda), I_{k}^{m \mp}(L(\lambda))\right\} .
$$

The following theorem is true:

Theorem 1. (i) The time flows given by the equations (8) are correctly defined and commute for all times $t_{k}^{m+}, t_{l}^{n-}$.

(ii) Euler-Arnold equations (8) are written in the "deformed" Lax form:

$$
\frac{\partial L(\lambda)}{\partial t_{k}^{m \mp}}=A(\lambda) M_{k}^{m \pm}(\lambda) L(\lambda)-L(\lambda) M_{k}^{m \pm}(\lambda) A(\lambda) .
$$

where $M_{k}^{m \pm}(\lambda)=\nabla I_{k}^{m}\left(L^{\mp}(\lambda)\right)=\sum_{j \in \mathbb{Z}_{ \pm}} \sum_{\alpha=1}^{\operatorname{dim} \mathfrak{g}_{\bar{j}}} \frac{\partial I_{k}^{m}}{\partial l_{\alpha}^{(j)}} X_{a}^{\bar{j}} \lambda^{j}$.

(iii) The functions $I_{q}^{p}\left(L^{ \pm}\right)$are constant along all times $t_{k}^{m \pm}$ and $t_{l}^{n \mp}$. 
The proof of this theorem repeats the proof of the analogous theorem for the case of ordinary loop algebras (see [3] and references therein).

Remark 4. Note that by virtue of the fact that functions $I_{k}^{m}\left(L^{\mp}(\lambda)\right)$ are finite polynomials $M$-operators $M_{k}^{m \pm}(\lambda)=\nabla I_{k}^{m}\left(L^{\mp}(\lambda)\right)$ belong to $\widetilde{\mathfrak{g}}_{A}^{\mathrm{pr} \pm}$, i.e. are finite linear combinations of the basic elements $X_{a}^{\bar{j}} \lambda^{j}$. This fact provides the correctness of the definition of equation (9).

\section{5 "Modified" Toda field equations}

\subsection{Zero-curvature condition with the values in $\widetilde{\mathfrak{g}}_{A}^{\mathrm{pr}}$}

In this subsection we will obtain zero curvature-type equations with the values in the Lie algeb$\operatorname{ras} \widetilde{\mathfrak{g}}_{A}^{\mathrm{pr}}$. The following theorem holds true:

Theorem 2. Let the infinite-dimensional Lie algebras $\widetilde{\mathfrak{g}}_{A}^{\mathrm{pr}}, \widetilde{\mathfrak{g}}_{A}^{\mathrm{pr}} \pm$, their dual spaces and polynomial Hamiltonians $I_{k}^{m}\left(L^{ \pm}(\lambda)\right), I_{s}^{n}\left(L^{ \pm}(\lambda)\right)$ on them be defined as in previous sections. Then the algebra-valued gradients of these functions satisfy the "deformed" zero-curvature equations:

$$
\begin{aligned}
& \frac{\partial \nabla I_{k}^{m}\left(L^{ \pm}(\lambda)\right)}{\partial t_{l}^{n \pm}}-\frac{\partial \nabla I_{s}^{n}\left(L^{ \pm}(\lambda)\right)}{\partial t_{k}^{m \pm}}+\left[\nabla I_{k}^{m}\left(L^{ \pm}(\lambda)\right), \nabla I_{s}^{n}\left(L^{ \pm}(\lambda)\right)\right]_{A(\lambda)}=0, \\
& \frac{\partial \nabla I_{k}^{m}\left(L^{ \pm}(\lambda)\right)}{\partial t_{l}^{n \mp}}-\frac{\partial \nabla I_{s}^{n}\left(L^{\mp}(\lambda)\right)}{\partial t_{k}^{m \pm}}+\left[\nabla I_{k}^{m}\left(L^{ \pm}(\lambda)\right), \nabla I_{s}^{n}\left(L^{\mp}(\lambda)\right)\right]_{A(\lambda)}=0 .
\end{aligned}
$$

Idea of the Proof. The statement of the theorem, i.e. validity of the equations (10), (11), follows from the commutativity of the "deformed" Lax flows constructed in the previous section.

Remark 5. Using the mentioned above realizations of $\widetilde{\mathfrak{g}}_{A}^{\mathrm{pr}}$ the "deformed" zero-curvature and Lax equations can be rewritten in the standard form, but in this case the corresponding $U-V$ and $L-M$ pairs will be more complicated and forcing us to work with the zero-curvature and Lax equations in the "deformed" form (10), (11).

Theorem 2 provides us with an infinite number of $\widetilde{\mathfrak{g}}_{A}^{\mathrm{pr}}$-valued $U-V$ pairs that satisfy zero curvature-type equations. The latter are non-linear equations in partial derivatives in the dynamical variables - matrix elements of the matrix $L(\lambda)$. In the terminology of [2] equations generated by the infinite set of $U-V$ pairs are called "integrable in the kinematic sense". In the next subsections we will consider the simplest examples of such integrable equations and their hierarchies.

\subsection{Modified Toda-field equations: general case}

In this subsection we obtain concrete examples of integrable equations satisfying the "deformed" zero-curvature representations constructed in the previous subsection. The most interesting of them will be a "modified" Toda field equations. We will construct these equations for all series of classical simple (reductive) lie algebras.

Let us at first consider a general situation. Let the "principal quasigraded" Lie algebra $\widetilde{\mathfrak{g}}_{A}^{\mathrm{pr}}$ and its decomposition $\widetilde{\mathfrak{g}}_{A}^{\mathrm{pr}}=\widetilde{\mathfrak{g}}_{A}^{\mathrm{pr}+}+\widetilde{\mathfrak{g}}_{A}^{\mathrm{pr}-}$ be defined as in the previous sections. Generic elements $L^{ \pm}(\lambda) \in\left(\widetilde{\mathfrak{g}}_{A}^{\mathrm{pr} \mp}\right)^{*}$ of the dual spaces have the following form:

$$
L^{+}(\lambda)=\lambda L^{(-1)}+\lambda^{2} L^{(-2)}+\cdots, \quad L^{-}(\lambda)=L^{(0)}+\lambda^{-1} L^{(1)}+\lambda^{-2} L^{(2)}+\cdots,
$$

where $L^{(k)} \in \mathfrak{g}_{-k}$. In particular $L^{(0)}=\sum_{i=1}^{n} l_{i}^{(0)} H_{i}, L^{(-1)}=\sum_{\alpha_{i} \in \Pi \bigcup-\Theta} l_{i}^{(-1)} E_{\alpha_{i}}$.

Now we can formulate the following theorem: 
Theorem 3. Let $\mathfrak{g}$ be one of the classical matrix Lie algebras $g l(n), \operatorname{so}(2 n+1), \operatorname{sp}(n)$ or so $(2 n)$. Let the "deformation" matrix A corresponding to the "principal" grading of $\mathfrak{g}$ be defined as in the Proposition 2. Then:

(i) among the corresponding equations (10) there is the equation equivalent to the following "Volterra coupled system":

$$
\partial_{t} u_{i}=u_{i} \beta_{i}^{A}(v), \quad \partial_{x} v_{i}=v_{i} \alpha_{i}(u),
$$

where $\beta_{i}^{A}$ are "pseudo-roots" on $\mathfrak{g}_{-1}$ defined by (5) and depending on the "deformation" matrix $A$ and Lie algebra $\mathfrak{g}, \alpha_{i} \in \Pi \bigcup\{-\Theta\}$ are linear forms (roots) on $\mathfrak{h}, H_{i}$ is the basis in $\mathfrak{h}, E_{-\alpha_{i}}$, $E_{\Theta}$ is the basis in $\mathfrak{g}_{-1}$, and

$$
U=u=\sum_{i=1}^{\operatorname{dim} \mathfrak{h}} u_{i} H_{i}, \quad V=\lambda^{-1} v=\lambda^{-1}\left(\sum_{\alpha_{i} \in \Pi \bigcup-\Theta} v_{i} E_{-\alpha_{i}}\right)
$$

is the corresponding $U-V$ pair.

(ii) Volterra coupled system (12) is written in the form of the "modified" Toda field equations:

$$
\partial_{x t}^{2} \psi_{i}=\partial_{x} \psi_{i}\left(\sum_{\alpha_{j} \in \Pi \cup\{-\Theta\}} \beta_{i, j}^{A} e^{\alpha_{j}(\psi)}\right),
$$

where $\psi=\sum_{i=1}^{n} \psi_{i} H_{i}$ and $\psi_{i}$ satisfy the following differential constraint $\partial_{x} \psi_{1} \partial_{x} \psi_{2} \cdots \partial_{x} \psi_{n}=c$.

Proof. To prove item (i) of the theorem it is enough to show that among the integrals $I_{k}^{m}$, $I_{l}^{n}$ are such integrals $I_{k_{0}}^{m_{0}}, I_{l_{0}}^{n_{0}}$ that $I_{k_{0}}^{m_{0}}\left(L^{+}(\lambda)\right) \equiv I_{k_{0}}^{m_{0}}\left(L^{(-1)}\right), I_{l_{0}}^{n_{0}}\left(L^{-}(\lambda)\right) \equiv I_{l_{0}}^{n_{0}}\left(L^{(0)}\right)$. Let us explicitly find these functions. Let us consider the generating series $I_{k_{0}}^{m_{0}}\left(L^{+}(\lambda)\right)$ :

$$
I_{k_{0}}^{m_{0}}\left(L^{+}(\lambda)\right)=I^{m_{0}}\left(A^{-1}(\lambda) L^{+}(\lambda)\right)=I^{m_{0}}\left(\left(1+A \lambda+A^{2} \lambda^{2}+\cdots\right)\left(\lambda L^{(-1)}+\lambda^{2} L^{(-2)}+\cdots\right)\right) .
$$

Taking into account that $I^{m_{0}}$ is a homogeneous polynomial on $\mathfrak{g}^{*}$ we easily obtain that $I_{m_{0}}^{m_{0}}\left(L^{+}(\lambda)\right)$ $=I_{m_{0}}^{m_{0}}\left(L^{(-1)}\right)$. Now, for the role of $I^{m_{0}}$ we have to take any Casimir function on $\mathfrak{g}$ that has a non-trivial restriction onto $\mathfrak{g}_{1}$. Such Casimir function always exists, because the generic element of the space $\mathfrak{g}_{\overline{1}}$ is never nilpotent.

Let us now consider the generating series $I^{n_{0}}\left(L^{-}(\lambda)\right)$. If we chose the generating Casimir function as follows: $I^{n_{0}}\left(L^{-}(\lambda)\right)=\operatorname{det} A(\lambda) \operatorname{Det}\left(L^{-}(\lambda) A(\lambda)^{-1}\right) \equiv \operatorname{Det} L^{-}(\lambda)$, we obtain:

$$
I_{0}^{n_{0}}\left(L^{-}(\lambda)\right)=\operatorname{Det}\left(L^{(0)}\right) .
$$

For such the Hamiltonians their matrix gradients have the following form: $\nabla I_{m_{0}}^{m_{0}}\left(L^{+}(\lambda)\right) \equiv$ $u=\sum_{i=1}^{n} u_{i} H_{i}, \nabla I_{0}^{n_{0}}\left(L^{-}(\lambda)\right) \equiv \lambda^{-1} v=\lambda^{-1}\left(\sum_{\alpha_{i} \in \Pi} v_{i} E_{-\alpha_{i}}+v_{0} E_{\Theta}\right)$, where $u_{i} \equiv \frac{\partial I_{m_{0}}^{m_{0}}}{\partial l_{i}^{(0)}}, v_{i} \equiv \frac{\partial I_{0}^{n_{0}}}{\partial l_{i}^{(-1)}}$. Substituting this into the corresponding zero-curvature condition (11) we obtain item (i) of the theorem.

Making the substitution of variables: $u_{i}=\partial_{x} \psi_{i}, v_{i}=e^{\alpha_{i}(\psi)}$ we obtain equation (13). Finally, taking into account the constancy of the Hamiltonian $I_{l_{0}}^{n_{0}}\left(L^{(0)}\right)$ along all time flows we obtain that $\prod_{i=1}^{n} u_{i}=$ const. That proves item (ii).

Remark 6. In $[22,23] g l(n)$ coupled Volterra systems were obtained using the Bäcklund transformation for the Toda integrable hierarchy. Such connection means that starting from the known integrable hierarchy and Bäcklund transformation one can generate a new integrable hierarchy (see [27]). The investigation of the possibility of such connection for the Volterra coupled systems, associated with the other series of classical matrix Lie algebras, is a separate problem and we will return to it in our subsequent publications. 


\subsection{Example: $g l(n)$-modified Toda field equation}

Let us consider the Lie algebra $\widetilde{g l(n)_{A}^{\mathrm{pr}}}$ defined in the Example 1 and its natural decomposition

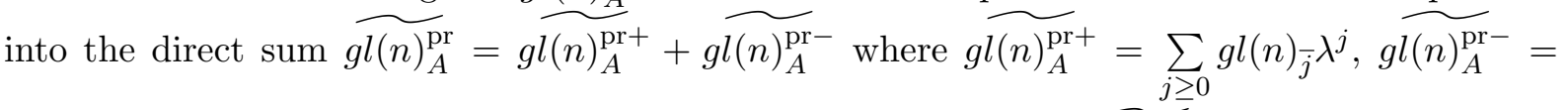
$\sum_{j<0} g l(n)_{j} \lambda^{j}$ and $\bar{j}=j \bmod n$. The generic elements $L^{ \pm}(\lambda) \in\left(\widetilde{g l(n)_{A}^{\mathrm{pr}} \mp}\right)^{*}$ of the dual spaces have the following form:

$$
L^{+}(\lambda)=\lambda L^{(-1)}+\lambda^{2} L^{(-2)}+\cdots, \quad L^{-}(\lambda)=L^{(0)}+\lambda^{-1} L^{(1)}+\lambda^{-2} L^{(2)}+\cdots,
$$

where $L^{(k)} \in \mathfrak{g}_{-k}: L^{(0)}=\sum_{i=1}^{n} l_{i}^{(0)} X_{i i}, L^{(-1)}=\sum_{i=1}^{n-1} l_{i}^{(-1)} X_{i, i+1}+l_{n}^{(-1)} X_{n 1}$. The simplest integrals are:

$$
D_{0}^{n-}(L(\lambda))=l_{1}^{(0)} l_{2}^{(0)} \cdots l_{n}^{(0)}, \quad D_{n}^{n+}(L(\lambda))=l_{1}^{(-1)} l_{2}^{(-1)} \cdots l_{n-1}^{(-1)} l_{n}^{(-1)} .
$$

Their matrix gradients yield the following $U-V$ pair:

$$
U \equiv \nabla D_{0}^{n-}=\sum_{i=1}^{n} u_{i} X_{i i}, \quad V \equiv \nabla D_{n}^{n+}=\lambda^{-1}\left(\sum_{i=1}^{n-1} v_{i} X_{i+1 i}+v_{n} X_{1 n}\right)
$$

where $u_{i} \equiv D_{0}^{n-} / l_{i}^{(0)}, v_{i} \equiv D_{n}^{n+} / l_{i}^{(-1)}$.

The corresponding "deformed" zero-curvature condition:

$$
\frac{\partial \nabla D_{0}^{n-}(L(\lambda))}{\partial t}-\frac{\partial \nabla D_{n}^{n+}(L(\lambda))}{\partial x}+\left[\nabla D_{0}^{n-}(L(\lambda)), \nabla D_{n}^{n+}(L(\lambda))\right]_{A(\lambda)}=0,
$$

yields the following equations ${ }^{2}$ :

$$
\begin{array}{ll}
\partial_{t} u_{1}=u_{1}\left(a_{1} v_{1}-a_{n} v_{n}\right), & \partial_{x} v_{1}=v_{1}\left(u_{2}-u_{1}\right), \\
\partial_{t} u_{2}=u_{2}\left(a_{2} v_{2}-a_{1} v_{1}\right), & \partial_{x} v_{2}=v_{2}\left(u_{3}-u_{2}\right), \\
\ldots \ldots \ldots \ldots \ldots \ldots \ldots \ldots \ldots \ldots \ldots \ldots \ldots \ldots & \ldots \ldots \ldots \ldots
\end{array}
$$

This system of the hyperbolic equations is the so-called "Volterra coupled systems". In the case of infinite $n$ and $a_{i} \equiv 1$ it was first considered in the paper [22]. In the non-periodic case it was also considered in the book [23].

This system goes together with the two constraints:

$$
u_{1} u_{2} \cdots u_{n}=\text { const }_{1}, \quad v_{1} v_{2} \cdots v_{n-1} v_{n}=\text { const }_{2},
$$

They follow from the constancy of the Hamiltonians $D_{0}^{n-}$ and $D_{n}^{n+}$ with respect to the all time flows.

In order for the "Volterra coupled systems" to acquire the form of the periodic "modified" Toda system it is enough to make the following change of variables [22]:

$$
u_{i}=\partial_{x} \psi_{i}, \quad i=1, n, \quad v_{i}=e^{\psi_{i+1}-\psi_{i}}, \quad i=1, n-1, \quad v_{n}=e^{\psi_{1}-\psi_{n}} .
$$

in the result we obtain the following system of equations:

$$
\partial_{x t}^{2} \psi_{1}=\partial_{x} \psi_{1}\left(a_{1} e^{\psi_{2}-\psi_{1}}-a_{n} e^{\psi_{1}-\psi_{n}}\right),
$$

\footnotetext{
${ }^{2}$ In the case $a_{i} \neq 0$ one may make $a_{i}=1$ rescaling the variables $v_{i}$.
} 


$$
\begin{aligned}
& \partial_{x t}^{2} \psi_{2}=\partial_{x} \psi_{2}\left(a_{2} \epsilon^{\psi_{3}-\psi_{2}}-a_{1} e^{\psi_{2}-\psi_{1}}\right) \\
& \cdots \cdots \cdots \cdots \cdots \cdots \cdots \cdots \cdots \cdots \cdots \cdots \cdots \cdots \cdots \cdots \cdots \cdots \cdots \\
& \partial_{x t}^{2} \psi_{n}=\partial_{x} \psi_{n}\left(a_{n} e^{\psi_{1}-\psi_{n}}-a_{n-1} e^{\psi_{n}-\psi_{n-1}}\right)
\end{aligned}
$$

with additional differential constraint:

$$
\partial_{x} \psi_{1} \partial_{x} \psi_{2} \cdots \partial_{x} \psi_{n}=c .
$$

The simplest case, when the corresponding constraint can be solved, is the case $\partial_{x} \psi_{i}=0$ for some fixed $i$. This is equivalent to the reduction $u_{i}=0$ in the corresponding Volterra coupled system. Let us put, for example, $\psi_{n}=$ const $=0$. In this case we obtain the following "open" $(n-1)$-component modified Toda chain:

$$
\begin{aligned}
& \partial_{x t}^{2} \psi_{1}=\partial_{x} \psi_{1}\left(a_{1} e^{\psi_{2}-\psi_{1}}-a_{n} e^{\psi_{1}}\right), \\
& \partial_{x t}^{2} \psi_{2}=\partial_{x} \psi_{2}\left(a_{2} \epsilon^{\psi_{3}-\psi_{2}}-a_{1} e^{\psi_{2}-\psi_{1}}\right), \\
& \cdots \cdots \cdots \cdots \cdots \cdots \cdots \cdots \cdots \cdots \cdots \cdots \cdots \cdots \cdots \cdots \cdots \cdots \cdots \cdots \cdots \cdots \\
& \partial_{x t}^{2} \psi_{n-1}=\partial_{x} \psi_{n-1}\left(a_{n-1} e^{-\psi_{n-1}}-a_{n-2} e^{\psi_{n-1}-\psi_{n-2}}\right) .
\end{aligned}
$$

\subsection{Example: $\mathfrak{g}=s o(2 n)$-modified Toda field equation}

Let us consider the Lie algebra $s \widetilde{(2 n)_{A}^{\mathrm{pr}}}$ being defined in the Example 4 and its natural decomposition into the direct sum $\widetilde{s o(2 n)_{A}^{\mathrm{pr}}}=s o \widetilde{(2 n)_{A}^{\mathrm{pr}}+}+s \widetilde{(2 n)_{A}^{\mathrm{pr}}-}$. Generic elements $L^{ \pm}(\lambda) \in$ $\left(s o(2 n)_{A}^{\mathrm{pr} \mp}\right)^{*}$ of the dual spaces have the following form:

$$
L^{+}(\lambda)=\lambda L^{(-1)}+\lambda^{2} L^{(-2)}+\cdots, \quad L^{-}(\lambda)=L^{(0)}+\lambda^{-1} L^{(1)}+\lambda^{-2} L^{(2)}+\cdots,
$$

where $L^{(k)} \in s o(2 n)_{-k} \bmod (2 n-2)$.

$$
\begin{aligned}
& L^{(0)}=\sum_{i=1}^{n} l_{i}^{(0)}\left(X_{i i}-X_{i+n i+n}\right), \\
& L^{(-1)}=\sum_{i=1}^{n-1} l_{i}^{(-1)}\left(X_{i i+1}-X_{i+n+1 i+n}\right)+l_{n}^{(-1)}\left(X_{n 2 n-1}-X_{n-12 n}\right)+l_{n+1}^{(-1)}\left(X_{n+12}-X_{n+21}\right) .
\end{aligned}
$$

It turned out that the form of the needed Casimir functions and Hamiltonians differs for the cases $n \leq 3$ and $n>3$. We will consider here only the case $n>3$. The simplest non-trivial integrals are:

$$
\begin{aligned}
& D_{0}^{2 n-}(L(\lambda))=\left(l_{1}^{(0)} l_{2}^{(0)} \cdots l_{n}^{(0)}\right)^{2} \\
& T_{2 n-2}^{(2 n-2)+}(L(\lambda))=l_{1}^{(-1)}\left(l_{2}^{(-1)}\right)^{2} \cdots\left(l_{n-2}^{(-1)}\right)^{2} l_{n-1}^{(-1)} l_{n}^{(-1)} l_{n+1}^{(-1)} .
\end{aligned}
$$

Their matrix gradients are:

$$
\begin{aligned}
U \equiv \nabla D_{0}^{2 n-}=\sum_{i=1}^{n} & u_{i}\left(X_{i i}-X_{i+n i+n}\right) \\
V \equiv \nabla T_{2 n-2}^{(2 n-2)+}= & \lambda^{-1}\left(\sum_{i=1}^{n-1} v_{i}\left(X_{i+1 i}-X_{i+n i+n+1}\right)+v_{n}\left(X_{2 n-1 n}-X_{2 n n-1}\right)\right. \\
& \left.+v_{n+1}\left(X_{2 n+1}-X_{1 n+2}\right)\right)
\end{aligned}
$$

where $u_{i} \equiv D_{0}^{2 n-} / l_{i}^{(0)}, v_{i} \equiv T_{2 n-2}^{(2 n-2)+} / l_{i}^{(-1)}$. 
The corresponding zero-curvature condition

$$
\frac{\partial \nabla D_{0}^{2 n-}(L(\lambda))}{\partial t}-\frac{\partial \nabla T_{2 n-2}^{(2 n-2)+}(L(\lambda))}{\partial x}+\left[\nabla D_{0}^{2 n-}(L(\lambda)), T_{2 n-2}^{(2 n-2)+}(L(\lambda))\right]_{A(\lambda)}=0,
$$

yields the following equations:

$$
\begin{array}{ll}
\partial_{t} u_{1}=u_{1}\left(a_{1} v_{1}-a_{n+1} v_{n+1}\right), & \partial_{x} v_{1}=v_{1}\left(u_{2}-u_{1}\right), \\
\partial_{t} u_{2}=u_{2}\left(a_{2} v_{2}-a_{1} v_{1}+a_{n+1} v_{n+1}\right), & \partial_{x} v_{2}=v_{2}\left(u_{3}-u_{2}\right), \\
\partial_{t} u_{3}=u_{3}\left(a_{3} v_{3}-a_{2} v_{2}\right), & \partial_{x} v_{3}=v_{3}\left(u_{4}-u_{3}\right), \\
\ldots \ldots \ldots \ldots \ldots \ldots \ldots \ldots \ldots \ldots \ldots \ldots \ldots \ldots \ldots \ldots \ldots \ldots \ldots \ldots \ldots \ldots \\
\partial_{t} u_{n-2}=u_{n-2}\left(a_{n-2} v_{n-2}-a_{n-3} v_{n-3}\right), & \ldots \ldots \ldots u_{n-1}\left(u_{n}-u_{n-1}\right), \\
\partial_{t} u_{n-1}=u_{n-1}\left(a_{n-1} v_{n-1}-a_{n-2} v_{n-2}-a_{n} v_{n}\right), & \partial_{x} v_{n-1}=v_{x} v_{n}=-v_{n}\left(u_{n}+u_{n-1}\right), \\
\partial_{t} u_{n}=u_{n}\left(a_{n} v_{n}-a_{n-1} v_{n-1}\right), & \partial_{x} v_{n+1}=v_{n+1}\left(u_{1}+u_{2}\right) .
\end{array}
$$

This system goes together with the natural constraints:

$$
u_{1} u_{2} \cdots u_{n}=\text { const }_{1}, \quad v_{1}\left(v_{2}\right)^{2} \cdots\left(v_{n-2}\right)^{2} v_{n-1} v_{n} v_{n+1}=\text { const }_{2} .
$$

which follows from the constancy of the Hamiltonians $D_{0}^{2 n-}$ and $T_{2 n-2}^{(2 n-2)+}$ with respect to the all time flows. Making the following replacement of variables:

$$
\begin{aligned}
& u_{i}=\partial_{x} \psi_{i}, \quad v_{i}=e^{\psi_{i+1}-\psi_{i}}, \quad \text { where } i=1, n-1, \\
& v_{n}=e^{-\left(\psi_{n+1}+\psi_{n}\right)}, \quad v_{n+1}=e^{-\left(\psi_{2}+\psi_{1}\right)}
\end{aligned}
$$

we obtain the $s o(2 n)$-modified Toda chain:

$$
\begin{aligned}
& \partial_{x t}^{2} \psi_{1}=\partial_{x} \psi_{1}\left(a_{1} e^{\psi_{2}-\psi_{1}}-a_{n+1} e^{\psi_{2}+\psi_{1}}\right), \\
& \partial_{x t}^{2} \psi_{2}=\partial_{x} \psi_{2}\left(a_{2} e^{\psi_{3}-\psi_{2}}-a_{1} e^{\psi_{2}-\psi_{1}}+a_{n+1} e^{\psi_{2}+\psi_{1}}\right), \\
& \partial_{x t}^{2} \psi_{3}=\partial_{x} \psi_{3}\left(a_{3} e^{\psi_{4}-\psi_{3}}-a_{2} e^{\psi_{3}-\psi_{2}}\right), \\
& \cdots \cdots \cdots \cdots \cdots \cdots \cdots \cdots \cdots \cdots \cdots \cdots \cdots \cdots \cdots \cdots \cdots \cdots \cdots \cdots \cdots \\
& \partial_{x t}^{2} \psi_{n-2}=\partial_{x} \psi_{n-2}\left(a_{n-2} e^{\psi_{n-1}-\psi_{n-2}}-a_{n-3} e^{\psi_{n-2}-\psi_{n-3}}\right), \\
& \partial_{x t}^{2} \psi_{n-1}=\partial_{x} \psi_{n-1}\left(a_{n-1} e^{\psi_{n}-\psi_{n-1}}-a_{n-2} e^{\psi_{n-1}-\psi_{n-2}}-a_{n} e^{-\left(\psi_{n-1}+\psi_{n}\right)}\right), \\
& \partial_{x t}^{2} \psi_{n}=\partial_{x} \psi_{n}\left(a_{n} e^{-\left(\psi_{n-1}+\psi_{n}\right)}-a_{n-1} e^{\psi_{n}-\psi_{n-1}}\right) .
\end{aligned}
$$

These equations goes also with additional differential constraint: $\partial_{x} \psi_{1} \partial_{x} \psi_{2} \cdots \partial_{x} \psi_{n}=c$.

\section{Conclusion and discussion}

In the present paper we have constructed a family of quasigraded Lie algebras that coincide with deformations of the "principal" subalgebras of the loop algebras and admit Kostant-AdlerSymes scheme. Using the constructed algebras we have obtained new Volterra coupled systems and modified Toda field equations for all series of classical matrix Lie algebras $\mathfrak{g}$.

Further, more detailed investigation of the constructed integrable hierarchies would be very interesting. In particular, an interesting open problem is to find a possible connection of the constructed new Volterra coupled systems and new modified Toda field equations with the Bäcklund transformations and the usual Toda field equations [19, 21, 23]. It would be also interesting to find explicit forms of the simplest equations of the "negative" and "positive" subhierarchies of the constructed integrable hierarchies in the case of general $\mathfrak{g}$. They should 
coincide with the "deformations" of the generalized mKdV equations [28] (see [29] where $g l(2)$ case was considered in details). It would be also very nice to find out whether there exists an analog of the "Drienfield-Sokolov" reduction [21] and the corresponding "deformed" KdV equations.

Another interesting development is to generalize the construction of the present paper onto the case of the other graded subalgebras of the loop algebras (see [20, 28]). The work in this direction is now in progress and the corresponding results will soon be published.

\section{Acknowledgements}

Author is grateful to P. Holod, S. Zykov and M. Pavlov for discussions. The research was supported by INTAS YS Fellowship Nr 03-55-2233 and by the French-Ukrainian project "Dnipro".

[1] Zaharov V., Shabat A., Integration of nonlinear equations of mathematical physics using the method of inverse scattering problem. II, Funktsional. Anal. i Prilozhen., 1979, V.13, N 3, 13-21 (in Russian).

[2] Takhtadzhyan L.A., Faddeev L.D., The Hamiltonian approach in soliton theory, Moscow, Nauka, 1986 (in Russian).

[3] Newell A., Solitons in mathematics and physics, University of Arizona, Society for Industrial and Applied Mathematics, 1985.

[4] Flaschka H., Newell A., Ratiu T., Kac-Moody Lie algebras and soliton equations. II. Lax equations associated with $A_{1}^{(1)}$, Phys. D, 1983, V.9, 303-323.

[5] Flaschka H., Newell A., Ratiu T., Kac-Moody Lie algebras and soliton equations. III. Stationary equations associated with $A_{1}^{(1)}$, Phys. D, 1983, V.9, 324-332.

[6] Holod P., Integrable Hamiltonian systems on the orbits of affine Lie groups and periodical problem for mKdV equation, Preprint ITF-82-144R, Kyiv, Institute for Theoretical Physics, 1982 (in Russian).

[7] Reyman A., Semenov-Tian-Shansky M., Group theoretical methods in the theory of finite-dimensional integrable systems, VINITI, Current Problems in Mathematics. Fundamental Directions, 1989, V.6, 145147 (in Russian).

[8] Holod P., Skrypnyk T., Anisotropic quasigraded Lie algebras on the algebraic curves and integrable Hamiltonian systems, Naukovi Zapysky NAUKMA, Ser. Phys.-Math. Sciences, 2000, V.18, 20-25 (in Ukrainian).

[9] Skrypnyk T., Lie algebras on algebraic curves and finite-dimensional integrable systems, nlin.SI-0010005 (talk on the XXIII International Colloquium on Group Theoretical Methods in Physics, July 31 - August 5, 2000, Dubna, Russia).

[10] Skrypnyk T., Quasigraded Lie algebras on hyperelliptic curves and classical integrable systems, J. Math. Phys., 2001, V.42, 4570-4581.

[11] Skrypnyk T., Quasigraded deformations of Lie algebras and finite-dimensional integrable systems, Czechoslovak J. Phys., 2002, V.52, 1283-1288.

[12] Skrypnyk T., Quasigraded Lie algebras and hierarchies of integrable equations, Czechoslovak J. Phys., 2003, V.53, 1119-1124.

[13] Golubchik I., Sokolov V., Compatible Lie brackets and integrable equations of the principle chiral model type, Funktsional. Anal. i Prilozhen., 2002, V.36, N 3, 9-19 (in Russian).

[14] Holod P., Hamiltonian systems connected with anisotropic affine Lie algebras and higher Landau-Lifshits equations, Dokl. Akad. Nauk Ukrain. SSR Ser. A, 1984, N 5, 5-8 (in Russian).

[15] Holod P., The hidden symmetry of the Landau-Lifshits equation, the hierarchy of higher equations and a dual equation for an asymmetric chiral field, Teoret. Mat. Fiz., 1987, V.70, N 1, 18-29 (in Russian).

[16] Skrypnyk T., Doubled generalized Landau-Lifshitz hierarchies and special quasigraded Lie algebras, J. Phys. A: Math. Gen., 2004, V.37, 7755-7768, nlin.SI/0403046.

[17] Skrypnyk T., Deformations of loop algebras and integrable systems: hierarchies of integrable equations, J. Math. Phys., 2004, V.45, 4578-4595.

[18] Skrypnyk T., Quasigraded Lie algebras, the Kostant-Adler scheme, and integrable hierarchies, Teoret. Mat. Fiz., 2005, V.142, N 2, 275-288 (in Russian).

[19] Mikhailov A., The reduction in integrable systems. Groups of reduction, Pis'ma Zh. Eksper. Teoret. Fiz., 1980, V.32, N 1, 187-192 (in Russian). 
[20] Kac V., Infinite-dimentional Lie algebras, Moscow, Mir, 1993 (in Russian).

[21] Drinfel'd V., Sokolov V., Lie algebras and KdV-type equations, VINITI, Current Problems in Mathematics. Fundamental Directions, 1984, V.24, 81-180 (in Russian).

[22] Shabat A., Yamilov R., To a transformation theory of two-dimensional integrable systems, Phys. Lett. A, 1997, V.277, 15-23.

[23] Leznov A., Saveliev M., Group methods for the integration of nonlinear dynamical systems, Moscow, Nauka, 1985 (in Russian).

[24] Krichiver I., Novikov S., Virasoro-type algebras, Riemannian surfaces and structures of the soliton theory, Funktsional. Anal. i Prilozhen., 1987, V.21, N 2, 46-64 (in Russian).

[25] Cantor I., Persits D., Closed stacks of Poisson brackets, in Proceedinds of the IX USSR Conference in Geometry, Kishinev, Shtinitsa, 1988, 141 (in Russian).

[26] Bolsinov A., Completeness of families of functions in involution that are connected with compatible Poisson brackets, Trudy Sem. Vektor. Tenzor. Anal., 1988, N 23, 18-38 (in Russian).

[27] Borisov A., Zykov S., The chain of the dressing discrete symmetries and generation of the non-linear equations, Teoret. Mat. Fiz., 1998, V.115, 199-214 (in Russian).

[28] de Groot M., Hollowood T., Miramontes J., Generalized Drienfield-Sokolov hierarchies, Comm. Math. Phys., 1992, V.145, 57-78.

[29] Skrypnyk T., Integrable deformations of the $\mathrm{mKdV}$ and $\mathrm{SG}$ hierarchies and quasigraded Lie algebras, Phys. D, to appear. 